Faura-Martínez, U., Lafuente-Lechuga, M. y Cifuentes-Faura, J. (2022). ¿Desigualdad territorial en Selectividad? Analizando la asignatura de matemáticas en Ciencias Sociales. Revista de Investigación Educativa,40(1), 69-87. DOI: http://dx.doi.org/10.6018/rie.424841

\title{
¿Desigualdad territorial en Selectividad? Analizando la asignatura de matemáticas en Ciencias Sociales
}

\section{Territorial inequality in Selectivity? Analyzing Mathematics in Social Sciences}

\author{
Úrsula Faura-Martínez, Matilde Lafuente-Lechuga' y Javier Cifuentes-Faura \\ *Departamento de Métodos Cuantitativos para la Economía. Facultad de Economía y Empresa. Universidad de Murcia
}

\section{Resumen}

Ante el debate sobre la posible desigualdad territorial en la Prueba de Acceso a la Universidad, conocida como Selectividad, este trabajo pretende arrojar un poco de luz a cerca de esta creciente preocupación.

Se analiza la estructura y resultados de las 17 pruebas autonómicas en la materia de Matemáticas Aplicadas a las Ciencias Sociales II, examinando el grado de dificultad de los ejercicios con la opinión de un panel de expertos.

Se detectan diferencias en la estructura del examen de acceso que han podido influir en los resultados obtenidos por el alumnado. A través del panel de expertos se constata el diferente grado de dificultad de los ejercicios y su posible correlación con la calificación media obtenida en cada región.

Los resultados evidencian la necesidad de llevar a cabo una revisión de la forma actual de las pruebas de acceso que elimine las desigualdades que puedan existir. Quizás habría que plantearse la realización de una única prueba a nivel nacional que garantice la igualdad en los contenidos y en los criterios de evaluación.

Palabras clave: Selectividad; Prueba de Acceso Universidad; Matemáticas Aplicadas Ciencias Sociales II; equidad; región.

1 Correspondencia: Matilde Lafuente Lechuga, mati@um.es, Facultad de Economía y Empresa, Campus de Espinardo, 30100 Murcia. 


\begin{abstract}
In view of the debate on the possible territorial inequality in the University Admissions Test, known as Selectivity, this paper aims to shed some light on this growing concern.

It analyses the structure and results of the 17 regional tests in the field of Mathematics Applied to Social Sciences II, examining the degree of difficulty of the exercises with the opinion of a panel of experts.

Differences in the structure of the admission tests have been detected that could have had an influence in the results obtained by the students. The panel of experts has noted the different degree of difficulty of the exercises and its possible correlation with the average mark obtained in each region.

The results demonstrate the need to review the current structure of access tests in order to eliminate the possible inequalities that may exist. It may be necessary to consider the development of only one test at a national level to ensure the equality of content and evaluation criteria.

Keywords: Selectivity; University Admissions Test; Mathematics Applied to Social Sciences II; equity; region.
\end{abstract}

\title{
Introducción
}

Para poder acceder a los estudios universitarios en España, los estudiantes deben realizar una prueba escrita denominada, desde 2017, Evaluación de Bachillerato para el Acceso a la Universidad, llamada por algunas comunidades autónomas como EBAU (Asturias, Canarias, Cantabria, Castilla y León, Extremadura, Región de Murcia, País Vasco y Rioja) y otras, como EvAU (Aragón, la Comunidad de Madrid, Castilla la Mancha y Navarra) prescindiendo del término Bachillerato, quedando como Evaluación de Acceso a la Universidad. En Andalucía se emplea el nombre de PEvAU, en Galicia, ABAU y en Baleares PBAU. Otras mantienen el nombre anterior, PAU, Pruebas de Acceso a la Universidad (Cataluña y Comunidad Valenciana). Esta prueba también es conocida como Selectividad (a partir de ahora se hace referencia a ella con este nombre).

El propósito de la Selectividad es valorar, de forma objetiva, los conocimientos y capacidades logrados por los estudiantes durante el bachillerato, su madurez académica y su capacidad para seguir con éxito las enseñanzas universitarias.

Fue reformada en 2010 y hasta entonces constaba de un mínimo de 20 exámenes escritos. En el curso 2009/2010 se establecen dos fases, la general y la específica que han evolucionado hasta el curso actual. La fase general es obligatoria para los que poseen el título de Bachillerato mientras que la fase específica es voluntaria y permite mejorar la calificación en los procesos de admisión a los estudios de Grado.

A pesar de su regulación a través de un Real Decreto existen diferencias en su estructura entre las distintas CCAA, al darle autonomía a estas. En la orden PCI/12/2019 (BOE, 2019), de 14 de enero, por la que se "determinan las características, el diseño y el contenido de la evaluación de Bachillerato para el acceso a la Universidad, y las fechas máximas de realización y de resolución de los procedimientos de revisión de las calificaciones obtenidas en el curso 2018-2019", en su artículo 5, establece que cada prueba constará de un número mínimo de 2 y un número máximo de 15 preguntas. También informa de que "tendrá una duración de un máximo de cuatro días, y aquellas 
Administraciones educativas con lengua cooficial podrán establecer una duración de un máximo de cinco días".

La simple diferencia de calendarios implica que se realicen 17 pruebas distintas (una por comunidad), con las posibles diferencias en sus criterios de corrección e incluso en los contenidos, aunque dentro del marco curricular de segundo curso de Bachillerato.

Cada curso académico, tras la realización de las pruebas, sobre todo en la convocatoria ordinaria, se abre el debate sobre el cambio del modelo actual para que exista un mismo grado de dificultad, aunque las pruebas no sean necesariamente iguales; o hay incluso quien va más allá al plantear que se realice una prueba común (al igual que en el examen de Médico Interno Residente-MIR) para asegurar la igualdad territorial. En el curso académico 2018-19 el debate se produjo con mayor intensidad a raíz de la prueba de Matemáticas II realizada en la Universidad de Valencia sobre la que más de 20000 alumnos firmaron una petición a la Conselleria de Educación para reclamar la anulación del examen por, según ellos, su excesiva complicación. Otros ejemplos de incidencias de esta convocatoria fue el protagonizado en la Universidad de Castilla la Mancha, dónde se tuvo que conceder 30 minutos más en la prueba de Matemáticas Aplicada a las Ciencias Sociales II (a partir de ahora MACSII) para resolver un problema. En Galicia se repitió el examen de Filosofía por la tarde, dado que ninguno de los seis exámenes disponibles estaba bien planteado. Estos son solo algunos ejemplos de incidencias, valoradas de manera muy distinta entre el alumnado, el profesorado y entre los padres y madres de los alumnos.

Por todo ello, se considera necesario, al menos, reflexionar sobre la disparidad de los exámenes y condiciones en la realización de las pruebas, y se han planteado estas cuestiones sobre una de las materias generales del bloque de asignaturas troncales de segundo curso de Bachillerato en Humanidades y Ciencias Sociales para el itinerario de Ciencias Sociales, MACSII. Ésta forma parte de la fase general, y por tanto obligatoria, de la Selectividad al ser una materia de modalidad en dicho Bachiller.

Se ha elegido esta asignatura, por diferentes razones. En primer lugar, porque tal y como aparece en el Real Decreto 1105/2014, de 26 de diciembre, (BOE, 2015) que establece el currículo básico de la Educación Secundaria Obligatoria y de Bachillerato, en el preámbulo de las MACSII, se destaca la importancia que éstas tienen en la formación intelectual de los estudiantes, su papel como herramienta para alcanzar y consolidar conocimiento, su capacidad para desarrollar el razonamiento y reflexionar sobre los temas sociales. Por otro lado, es una asignatura cuya corrección es bastante objetiva y fácilmente comparable y porque los autores de este trabajo son especialistas en esta materia.

Se plantea, en este trabajo, un doble objetivo. Por un lado, analizar la estructura de las 17 pruebas respecto a la distribución horaria, el número de ejercicios a realizar y la puntuación asignada a cada uno de ellos, entre otras, $\mathrm{y}$, en segundo lugar, analizar el rendimiento académico de los estudiantes, por CCAA; en la convocatoria ordinaria de 2018, relacionándolo con la opinión dada por un panel de expertos en la materia MACSII sobre la dificultad de estos exámenes. 


\section{Revisión de la Literatura}

Las investigaciones realizadas sobre las pruebas de acceso a la Universidad se centran fundamentalmente en dos líneas. Por un lado, las que cuestionan su conveniencia, apoyan la necesidad de cambios en su estructura, realización de una única prueba o varias dependiendo de los estudios a los que se quiera acceder, fiabilidad y objetividad de las pruebas, etc. La otra línea versa sobre la relación entre los resultados de la prueba y distintas variables como sexo, expediente del alumno, tipo de centro donde se ha cursado el bachillerato, etc.

Entre los primeros trabajos sobre esta temática ${ }^{2}$ está el de Bernad y Escudero (1978) destacando que el factor tribunal introduce sesgos en la prueba. Escudero y Bueno (1994) explican una experiencia promovida y financiada por el Ministerio de Educación que se llevó a cabo en la Universidad de Zaragoza consistente en la creación de un tribunal paralelo al oficial para contrastar si existían diferencias entre tribunales en las pruebas de acceso en junio de 1992. Concluyeron que "Los hallazgos de este estudio del doble tribunal en la selectividad son, sobre todo, una demostración de que nuestro sistema de selección de universitarios tiene algunos problemas de consistencia entre tribunales, pero ni mucho menos es una especie de lotería según el tribunal que toque" (Escudero y Bueno, 1994, p.296).

Diferentes estudios (Muñoz-Repiso y Murillo-Torrecilla, 1997; Cuxart y Longford, 1998; Gairin, Muñoz y Oller, 2012) apuntan que existen diferencias entre las pruebas realizadas, siendo una causa de las posibles diferencias el utilizar distintos criterios a la hora de corregir el examen. Otros proponen distintas actuaciones para que el resultado sea más fiable (Gairin, Muñoz y Oller, 2013).

Lorenzo, Argos, Hernández y Vera (2014) plantean una serie de preguntas conducentes a conocer si la prueba de acceso cumple con las funciones inicialmente establecidas: ¿sirve para evaluar la madurez del estudiante ante su paso a la Universidad?, ¿sirve para homologar y certificar los conocimientos adquiridos en el Bachillerato?, ¿sirve para ubicar al estudiante correctamente, ordenarlo y que tenga prioridad en la elección de su grado? Concluyen que, de forma efectiva, solo se cumple esta última pregunta y proponen realizar una única prueba específica para ingresar en titulaciones con límite de plazas.

Aunque las pruebas de acceso a la universidad han recibido una creciente atención por parte de la comunidad educativa durante la primera década del siglo XXI (Grau, Cuxart y Martí, 2002; Gaviria, 2005; Contreras, Ordóñez y Wilhelmi, 2010; Ruiz de Gauna, Dávila, Etxeberria, y Sarasua, 2013; Ruiz-Hidalgo, Herrera y Velasco, 2019), son escasos los trabajos que se han realizado sobre la materia de MACSII y se centran en el método de evaluación y en el tipo de ejercicios, y no en las diferencias de las pruebas por CCAA. Ruiz de Gauna, Sarasua y García (2011) analizan el examen en el País Vasco y afirman que es la asignatura con mayor índice de suspensos de la prueba de acceso tanto a nivel de su región cómo del conjunto nacional y estudian la tipología de los ejercicios para poder mejorar los resultados. Boal, Bueno, Lerís y

2 Para una revisión detallada de los primeros estudios sobre las pruebas de acceso a la Universidad en España, véase Muñoz-Repiso, Muñoz, Palacios y Valle (1991). 
Sein-(2008) analizan las habilidades matemáticas evaluadas en las Pruebas de Acceso a la Universidad. En Nortes, Nortes y Lozano (2015) consideran las correcciones realizadas por dos evaluadores en el examen de junio de 2014 en la Universidad de Murcia para constatar que no existen diferencias significativas en los resultados obtenidos por ellos. En Rodríguez-Muñiz, Díaz, Mier y Alonso (2016) se indaga sobre el tipo de problemas propuestos en el examen de MACSII en cuatro Universidades (Andalucía, Asturias, Madrid y el País Vasco) y su relación con el currículo oficial. En Mengual, Albarracín, Muñoz-Escolano, Oller-Marcén, y Gorgorió (2019), se propone un proceso para construir criterios que permita calificar exámenes de matemáticas reduciendo la variabilidad que pudiera existir por parte de correctores diferentes.

\section{Metodología}

Se ha realizado un estudio descriptivo de la estructura de las pruebas de Selectividad de las 17 CCAA, analizando el desarrollo temporal de la misma, la puntuación de cada ejercicio, si los ejercicios son obligatorios o pueden elegir entre varios, si se permite el uso de calculadora.... Se han recopilado todas las pruebas de MACSII, en la convocatoria ordinaria de 2018, de las distintas Comunidades Autónomas. Las relativas a las dos ciudades autónomas, Ceuta y Melilla, al depender de la Universidad de Granada, están incluidas en Andalucía.

En el curso académico 2015-2016 llega a la Prueba de acceso a la Universidad la Ley Orgánica 8/2013, de 9 de diciembre, para la mejora de la calidad educativa (LOMCE), denominada popularmente como "ley Wert", lo que supuso cambios en los contenidos y ponderaciones de esta prueba. Por ello, se ha estudiado la evolución de los resultados, tanto globales como de la materia MACSII de tres cursos académicos afectados por este cambio de ley, centrándose el estudio, más detalladamente, en el curso 2017-2018.

Para contrastar los resultados en las distintas pruebas de MACSII con su posible nivel de dificultad, se ha elaborado una plantilla para que un panel de expertos de profesores, tanto universitarios como de enseñanzas medias, vinculados a la Selectividad y que han formado parte, alguna vez, del equipo de correctores de las pruebas de acceso en la materia de MACSII, valoren la complejidad de cada examen.

La composición de este panel parte de una base de datos formada por compañeros de todas las CCAA que han sido miembros recientemente de los tribunales de corrección de esta asignatura y, por tanto, conocedores de la materia y de las pruebas. Mediante un correo electrónico se les informó de este proyecto argumentándoles la necesidad de contar con su colaboración para reflejar su opinión sobre las pruebas de acceso. En febrero de 2019 teníamos ya dos representantes de cada región que pasaron a formar el panel de expertos encargado de determinar el mayor o menor grado de dificultad de los ejercicios.

Con la ayuda de los expertos se elaboraron las rúbricas (tabla 1) para determinar el grado de dificultad de los ejercicios. A los profesores se les proporcionó los enunciados de los exámenes de MACSII, de la convocatoria ordinaria de 2018, de todas las CCAA, excepto el de su propia región, en la primera semana de abril, con el compromiso de que contestaran en un plazo máximo de 10 días. Además, debían rellenar una plantilla, en Excel, que le proporcionamos, en la que tenían que especificar el nivel de dificultad 
encontrado en cada ejercicio, valorado de 1 (muy fácil) a 10 (muy difícil). Los apartados de los problemas de la prueba se puntúan teniendo en cuenta las rúbricas de la tabla 1. La puntuación final de la dificultad de un examen dada por el docente, será la media ponderada por la puntuación reflejada en cada apartado de las distintas valoraciones de los apartados del examen.

Todos los expertos han examinado 16 pruebas (una por región, excepto la suya) y, por tanto, cada una ha sido puntuada 32 veces. Con esta información se ha asignado a un examen, como valoración del grado de dificultad, la puntuación media de estos datos. Se ha analizado la fiabilidad de las evaluaciones de los expertos a través del coeficiente alpha de Cronbach ${ }^{3}$. Un valor de este coeficiente superior a 0,7 revela una fuerte relación.

Los apartados de los problemas de la prueba se puntúan teniendo en cuenta las rúbricas de la tabla 1 .

Tabla 1.

Rúbricas para determinar el nivel de dificultad de un ejercicio de matemáticas

\begin{tabular}{cl}
\hline Nivel de dificultad & \multicolumn{1}{c}{ Rúbrica } \\
$\mathbf{1}-\mathbf{2}$ & $\begin{array}{l}\text { Enunciados sencillos con resolución de los ejercicios inmediata. } \\
\text { Enunciados sencillos con resolución de los ejercicios que requieren cál- } \\
\text { culos intermedios. }\end{array}$ \\
$\mathbf{5 - 6}$ & $\begin{array}{l}\text { Enunciado elaborado con resolución de los ejercicios empleando con- } \\
\text { ceptos inmediatos. } \\
\text { Enunciado elaborado con resolución de los ejercicios que implican do- } \\
\text { minar con bastante destreza los cálculos y propiedades de los concep- } \\
\text { tos que se está evaluando. } \\
\text { Enunciado complicado de entender con resolución de los ejercicios uti- } \\
\text { lizando conocimientos elevados de la materia. }\end{array}$ \\
\hline
\end{tabular}

\section{Estructura del examen de MACSII}

Las características, el diseño, así como el contenido de la prueba de acceso a la Universidad para el curso 2018-2019 se recogen en el artículo 8 de la Orden PCI/12/2019 de 14 de enero. También se incluyen las fechas máximas de realización y de resolución de los procedimientos de revisión de los resultados. Esta ley indica:

“En la elaboración de cada una de las pruebas de la evaluación se procurará utilizar al menos un estándar de aprendizaje por cada uno de los bloques de contenido, o agrupaciones de los mismos, que figuran en la matriz de especificaciones de la materia correspondiente" (BOE, 2019, p.2693).

3 Para calcular este coeficiente, como cada experto evalúa a todas las CCA excepto a la suya, los datos faltantes han sido imputados por la media correspondiente. 
Para la materia MACSII la matriz de especificaciones, recogida en el anexo I del artículo mencionado anteriormente (pp. 2693-2694), está dividida en cuatro bloques de contenidos:

- Bloque 1. Procesos, métodos y actitudes en matemáticas con un porcentaje asignado del $20 \%$ sobre la calificación de la prueba.

- Bloque 2. Números y Álgebra, con un 25\% asignado.

- Bloque 3. Análisis, con un $25 \%$ de porcentaje.

- Bloque 4. Estadística y Probabilidad, con un 30\% de ponderación.

Estas ponderaciones asignadas a cada uno de los bloques son orientativas. Al menos el $70 \%$ de los contenidos evaluables los establece el Estado con la matriz de especificaciones y son las comunidades autónomas las que determinan el 30\% restante. Las diferencias observables entre regiones podrían ser derivadas de ese $30 \%$ que cada autonomía establece o por el nivel de dificultad diferente en la prueba.

En la tabla 2 se recogen las características principales de la prueba en las distintas CCAA. En la convocatoria de junio de 2018, el examen se celebró en todas las regiones durante tres días en horario de mañana y tarde salvo en Andalucía que se realiza solo por las mañanas (de 8 a 15 horas) y Galicia y el País Vasco que la efectúan en dos días y medio. El examen de la materia Matemáticas Aplicadas se realiza el segundo día en todas las CCAA salvo en Cataluña, la C. Valenciana y el País Vasco que se examinan el primer día y Aragón y Baleares que se deja para el tercer día por la mañana. (tabla 2)

El uso de la calculadora durante la prueba de MACSII está restringido, en la mayoría de autonomías, a aquellas que no sean programables y que no tengan pantalla gráfica. No deben tener la opción de calcular derivadas, ni integrales, ni determinantes; asimismo, no deben poder resolver ecuaciones, ni realizar operaciones con matrices. Sin embargo, en cuatro regiones (Baleares, Cataluña, Castilla-La Mancha y Canarias) se permite cualquier tipo de calculadora.

Tabla 2.

Características de la prueba por Comunidades Autónomas

\begin{tabular}{|c|c|c|c|c|}
\hline CCAA & Nombre & Días & $\begin{array}{l}\text { Ubicación } \\
\text { temporal } \\
\text { MATCSII }\end{array}$ & $\begin{array}{c}\text { Contenido } \\
\text { Ejercicios (puntuación) }\end{array}$ \\
\hline Andalucía & PEvAU & $\begin{array}{l}3 \text { días en } \\
\text { horario } \\
\text { de ma- } \\
\text { ñana }\end{array}$ & Segundo día & $\begin{array}{l}\text { - } 1 \text { de Números y Álgebra (2.5) } \\
\text { - } 1 \text { de Análisis (2.5) } \\
\text { - } 1 \text { de Probabilidad (2.5) } \\
\text { - } 1 \text { de Inferencia y Muestreo (2.5) }\end{array}$ \\
\hline Aragón & EvAU & 3 días & $\begin{array}{l}\text { Tercer día - } \\
\text { mañana }\end{array}$ & $\begin{array}{l}\text { - } 1 \text { de Números y Álgebra (3.25) } \\
\text { - } 1 \text { de Análisis (3.25) } \\
\text { - } 1 \text { de Estadística y Probabilidad (3.5) }\end{array}$ \\
\hline
\end{tabular}




\begin{tabular}{|c|c|c|c|c|}
\hline CCAA & Nombre & Días & $\begin{array}{l}\text { Ubicación } \\
\text { temporal } \\
\text { MATCSII } \\
\end{array}$ & $\begin{array}{c}\text { Contenido } \\
\text { Ejercicios (puntuación) }\end{array}$ \\
\hline Asturias & EBAU & $\begin{array}{l}2 \text { días y } \\
\text { medio }\end{array}$ & $\begin{array}{l}\text { Segundo día } \\
\text { - mañana }\end{array}$ & $\begin{array}{l}\text { - } 1 \text { de Números y Álgebra (3) } \\
\text { - } 1 \text { de Análisis (3) } \\
\text { - } 1 \text { de Probabilidad (2) } \\
\text { - } 1 \text { de Inferencia y Muestreo (2) }\end{array}$ \\
\hline Balears & PBAU & 3 días & $\begin{array}{l}\text { Tercer día - } \\
\text { mañana }\end{array}$ & $\begin{array}{l}\text { - } 1 \text { de Números y Álgebra (2.5) } \\
\text { - } 1 \text { de Análisis (2.5) } \\
\text { - } 1 \text { de Estadística y Probabilidad (2.5) } \\
\text { - Una de las opciones tiene un ejercicio } \\
\text { más de Estadística y Probabilidad (2.5) } \\
\text { y la otra, un ejercicio más de Números } \\
\text { y Algebra o de Análisis (2.5) }\end{array}$ \\
\hline Canarias & EBAU & 3 días & $\begin{array}{l}\text { Segundo día } \\
\text { - mañana }\end{array}$ & $\begin{array}{l}\text { - } 1 \text { de Números y Álgebra (2.5) } \\
\text { - } 1 \text { de Análisis (2.5) } \\
\text { - } 1 \text { de Probabilidad (2.5) } \\
\text { - } 1 \text { de Inferencia y Muestreo (2.5) } \\
\end{array}$ \\
\hline Cantabria & EBAU & 3 días & $\begin{array}{l}\text { Segundo día } \\
\text { - mañana }\end{array}$ & $\begin{array}{l}\text { - } 1 \text { de Álgebra (3.5) } \\
\text { - } 1 \text { de Análisis (3.5) } \\
\text { - } 1 \text { de Probabilidad y Estadística (3). }\end{array}$ \\
\hline $\begin{array}{l}\text { Castilla - La } \\
\text { Mancha }\end{array}$ & EvAU & 3 días & $\begin{array}{l}\text { Segundo día } \\
\text { - mañana }\end{array}$ & $\begin{array}{l}\text { - } 2 \text { de Algebra }(1.5+2) \\
\text { - } 2 \text { de Análisis }(1.5+1.5) \\
\text { - } 1 \text { de Estadística }(2) \\
\text { - } 1 \text { de probabilidad }(1.5) \\
\end{array}$ \\
\hline $\begin{array}{l}\text { Castilla y } \\
\text { León }\end{array}$ & EBAU & $\begin{array}{l}2 \text { días y } \\
\text { medio }\end{array}$ & $\begin{array}{l}\text { Segundo día } \\
\text { - mañana }\end{array}$ & $\begin{array}{l}\text { - } 1 \text { de Álgebra (3) } \\
\text { - } 1 \text { de Análisis (3) } \\
\text { - } 1 \text { de Probabilidad (3) } \\
\text { - } 1 \text { de Estadística (1) } \\
\end{array}$ \\
\hline Cataluña & PAU & 3 días & $\begin{array}{l}\text { Primer día - } \\
\text { mañana }\end{array}$ & $\begin{array}{l}6 \text { cuestiones de las que hay que responder } \\
\text { 5. } \\
\text { - } 3 \text { de algebra y optimización. } \\
\text { - } 3 \text { de análisis. } \\
\text { Cada pregunta vale } 2 \text { puntos. }\end{array}$ \\
\hline $\begin{array}{l}\text { Comunitat } \\
\text { Valenciana }\end{array}$ & PAU & 3 días & $\begin{array}{l}\text { Primer día - } \\
\text { tarde }\end{array}$ & $\begin{array}{l}\text { - } 1 \text { de Números y Álgebra } \\
\text { - } 1 \text { de Análisis } \\
\text { - } 1 \text { de Estadística y Probabilidad } \\
\text { Todos con la misma puntuación. }\end{array}$ \\
\hline Extremadura & EBAU & 3 días & $\begin{array}{l}\text { Segundo día } \\
\text { - tarde }\end{array}$ & $\begin{array}{l}\text { - } 1 \text { de Números y Álgebra } \\
\text { - } 1 \text { de Análisis } \\
\text { - } 1 \text { de Estadística y Probabilidad } \\
\text { Todos con la misma puntuación. } \\
\text { Las preguntas serán contextualizadas siem- } \\
\text { pre que sea posible }\end{array}$ \\
\hline
\end{tabular}




\begin{tabular}{|c|c|c|c|c|}
\hline CCAA & Nombre & Días & $\begin{array}{l}\text { Ubicación } \\
\text { temporal } \\
\text { MATCSII } \\
\end{array}$ & $\begin{array}{c}\text { Contenido } \\
\text { Ejercicios (puntuación) }\end{array}$ \\
\hline Galicia & ABAU & $\begin{array}{l}2 \text { días y } \\
\text { medio }\end{array}$ & $\begin{array}{l}\text { Segundo día } \\
\text { - mañana }\end{array}$ & $\begin{array}{l}\text { - } 1 \text { de Números y Álgebra } \\
\text { - } 1 \text { de Análisis } \\
\text { - } 1 \text { de Estadística y Probabilidad } \\
\text { Todos con la misma puntuación. }\end{array}$ \\
\hline Madrid & EvAU & 3 días & $\begin{array}{l}\text { Segundo día } \\
\text { - mañana }\end{array}$ & $\begin{array}{l}\text { Una de las opciones contendrá } \\
2 \text { ejercicios de Números y Álgebra } \\
\text { - } 1 \text { de Análisis } \\
\text { - } 2 \text { de Estadística. } \\
\text { La otra opción contendrá } \\
\text { - } 1 \text { de Números y Álgebra } \\
\text { - } 2 \text { de Análisis } \\
\text { - } 2 \text { de Estadística y probabilidad } \\
\text { Todos con la misma puntuación. }\end{array}$ \\
\hline Murcia & EBAU & 3 días & $\begin{array}{l}\text { Segundo día } \\
\text { - mañana }\end{array}$ & $\begin{array}{l}5 \text { ejercicios repartidos: Los ejercicios de } \\
\text { Números y Álgebra con un peso entre el } \\
30 \% \text { y el } 35 \% \text {, los de Análisis con un peso } \\
\text { entre el } 30 \% \text { y el } 40 \% \text { y los de Estadística y } \\
\text { Probabilidad entre el } 30 \% \text { y } 40 \% \text {. }\end{array}$ \\
\hline Navarra & EvAU & 3 días & $\begin{array}{l}\text { Segundo día } \\
\text { - tarde }\end{array}$ & $\begin{array}{l}\text { - } 1 \text { de Números y Álgebra (3.5) } \\
\text { - } 1 \text { de Análisis (3.5) } \\
\text { - } 1 \text { de Estadística y Probabilidad (3) }\end{array}$ \\
\hline País Vasco & EBAU & $\begin{array}{l}2 \text { días y } \\
\text { medio }\end{array}$ & $\begin{array}{l}\text { Primer día - } \\
\text { tarde }\end{array}$ & $\begin{array}{l}\text { - } 1 \text { de Números y Álgebra (3) } \\
\text { - } 1 \text { de Análisis (3) } \\
\text { - } 1 \text { de Probabilidad (2) } \\
\text { - } 1 \text { de Muestreo (2) } \\
\end{array}$ \\
\hline Rioja & EBAU & 3 días & $\begin{array}{l}\text { Segundo día } \\
\text { - tarde }\end{array}$ & $\begin{array}{l}5 \text { ejercicios: } 3 \text { obligatorios más } 3 \text { ejercicios } \\
\text { para escoger } 2\end{array}$ \\
\hline
\end{tabular}

La estructura del examen de MACSII varía entre las distintas CCAA. En todas ellas siempre aparecen dos opciones, pero el número de ejercicios cambia de unas a otras y la puntuación de los mismos también.

En Andalucía, Baleares y Canarias la prueba consta de 4 ejercicios con la misma puntuación (en el caso de Baleares, se puntúa cada pregunta sobre 10 puntos): el primero de Números y Álgebra, el segundo de Análisis; el tercero es un ejercicio de Probabilidad, y el último de Inferencia y Muestreo.

En Galicia, el Principado de Asturias y el País Vasco hay 4 ejercicios: un ejercicio de Números y Álgebra (puntuación máxima de 3 puntos), un ejercicio de Análisis (hasta 3 puntos), un ejercicio de Probabilidad valorado con 2 puntos y el último de Inferencia y Muestreo ( 2 puntos). En Castilla y León la distribución es la misma, pero varía la puntuación (3, 3, 3 y 1 punto, respectivamente). 
En Aragón, Cantabria, la Comunidad Valenciana (cada ejercicio se valora con 10 puntos), Extremadura y Navarra el examen consta de tres preguntas, la primera del bloque Números y Álgebra, la segunda del bloque Análisis y la última del bloque Estadística y Probabilidad, pero la puntuación asignada a cada uno de los bloques es diferente.

En Castilla la Mancha se realizan 6 ejercicios: dos de Algebra (1.5+2), dos de Análisis $(1.5+1.5)$, uno de Probabilidad (1.5 puntos) y otro de Estadística (2 puntos).

En Cataluña aparecen 6 cuestiones de las que hay que responder 5. Cada pregunta vale 2 puntos. Hay 3 del Bloque 2, Números y Álgebra, y 3 del Bloque de Análisis. No hay integrales, ni probabilidad ni estadística.

En la Comunidad de Madrid se realizan 5 ejercicios, todos con igual puntuación. En cada opción la distribución es distinta. Una de ellas contiene dos ejercicios correspondientes al Bloque 2 (Números y Álgebra), uno al Bloque 3 (Análisis) y dos Bloque 4 (Estadística y Probabilidad). En la otra opción hay un ejercicio correspondiente al Bloque 2, dos al Bloque 3 y dos del 4 .

En la Región de Murcia hay 5 ejercicios con la siguiente distribución: el bloque de Números y Álgebra con un peso entre el $30 \%$ y el $35 \%$, el de Análisis con un peso entre el 30\% y el $40 \%$ y el de Estadística y Probabilidad entre el 30\% y $40 \%$.

En La Rioja hay 3 ejercicios obligatorios más otros 3 de los que hay que escoger 2; cada uno de los ejercicios vale 2 puntos. Los ejercicios obligatorios son iguales en las dos opciones.

\section{Resultados}

En este apartado se muestran los resultados finales obtenidos por el alumnado en la prueba de acceso a la Universidad en la convocatoria de junio del curso 2017-2018 en cada CCAA y en la materia de MACSII. De igual forma se recoge la opinión del panel de expertos sobre el grado de dificultad de esta materia.

\section{La Prueba de Acceso a la Universidad por comunidades autónomas en la convoca- toria ordinaria 2018}

Según los datos publicados por el Ministerio de Educación y Ciencia, el 93.29\% de los estudiantes aprobaron la prueba de selectividad de 2018 en la fase general de la categoría genérica en la convocatoria ordinaria (en junio) mientras que en la extraordinaria (julio o septiembre dependiendo de la CCAA) fue del $75.40 \%$, datos muy similares a los obtenidos en los últimos años.

Por CCAA, el resultado más bajo en la convocatoria de junio lo tiene Galicia con un $89.93 \%$ de los estudiantes presentados y el más alto le corresponde al País Vasco con un $97.90 \%$.

Como se aprecia en la figura 1 , donde se recoge el porcentaje de aprobados en Selectividad desde el curso 2015-2016 hasta el 2017-2018 por CCAA, los resultados son dispares. Hay regiones que presentan una mayor variabilidad, como es el caso de Asturias, que en 2016 tenía un 83.61\% de aprobados frente al 91.11\% en 2018. Otras como Aragón o Navarra tienen un porcentaje de aprobados similar en todos los años, alrededor del 95\%. En Baleares, el porcentaje de aprobados ha descendido en los dos 
últimos años en poco menos de 5 puntos. En general, la desviación típica existente en cada CCAA está alrededor de un punto (en bastantes casos muy por debajo), salvo en el caso ya comentado de Asturias dónde se llega a 3.8 puntos.

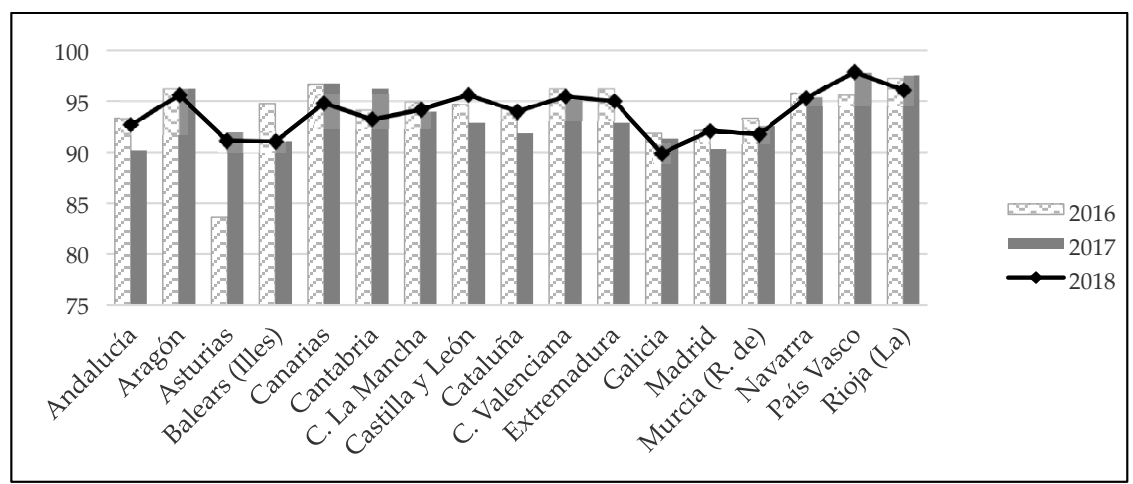

Figura 1. Porcentaje de aprobados en selectividad sobre presentados en la convocatoria ordinaria

En la figura 2 se presenta la nota media de la prueba de Selectividad para las diferentes CCAA en el año 2018. La más elevada corresponde a Extremadura, cerca de un 7, estando la nota media nacional en 6.49. Baleares es la región con los peores resultados, alejándose casi un punto de esta media, siendo su calificación un 16\% inferior a la de Extremadura. Por debajo de la calificación media se encuentran solo 7 regiones, 4 de ellas (Castilla la Mancha, Cantabria, Andalucía y Madrid) con valores muy cercanos a este promedio.

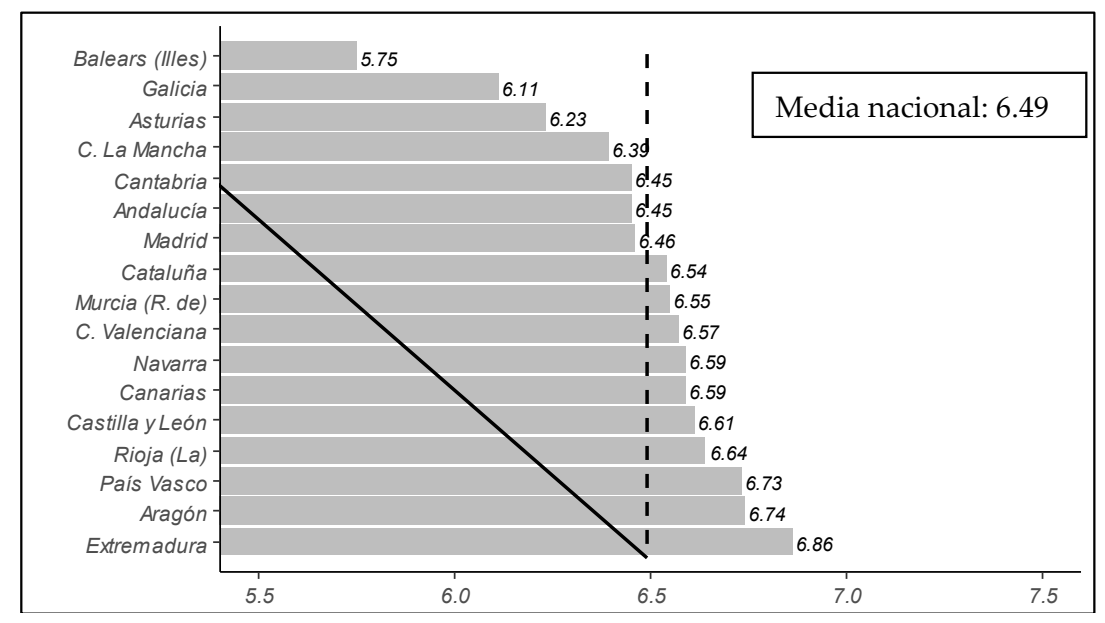

Figura 2. Nota media de Selectividad en 2018 en la convocatoria ordinaria

Los resultados alcanzados por los estudiantes en el periodo analizado en la asignatura de MACSII se recogen en la figura 3. La mayor media anual nacional en esta 
materia se alcanza en el año 2015, cercana a 7 puntos, con una desviación típica de 0.9. En el año 2017 existe menor dispersión entre las distintas regiones; variando todas en torno a un punto alrededor de la media nacional (6.,05 puntos), salvo en el caso de Canarias que no llega al aprobado. Fijándonos en cada una de las comunidades se observa que hay algunas dónde la media se ha mantenido en este periodo, como es el caso de Castilla la Mancha y otras dónde la tendencia ha ido decreciendo (Baleares, Cataluña). En la mayoría de ellas existe bastante dispersión (destacan la Comunidad Valenciana y el País Vasco). La tasa media de variación en el periodo estudiado muestra un crecimiento positivo solo en cinco regiones (Andalucía, Canarias, Castilla y León, Castilla la Mancha y Madrid) y el mayor decrecimiento se produce en el País Vasco con una caída media de poco más del 10\%.

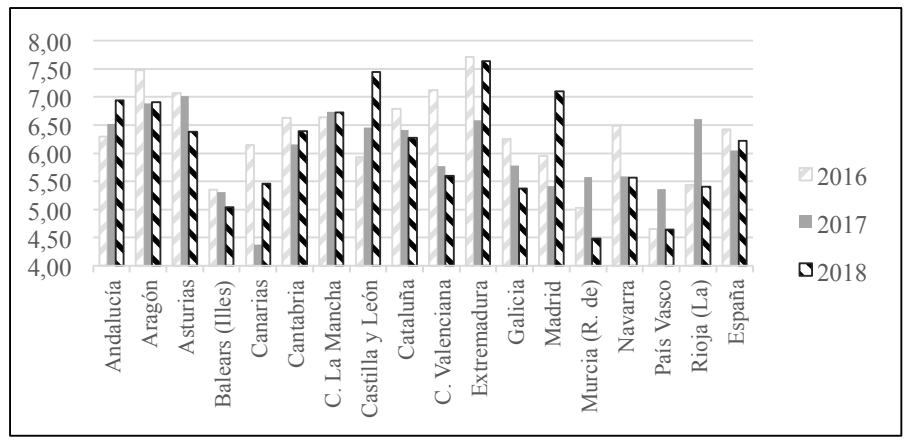

Figura 3. Nota media de MACSII

En 2018 (figura 4), los estudiantes de la Región de Murcia y del País Vasco tienen una calificación media inferior a 5 puntos. Por encima de la nota media de España, que se alcanza con 6.22 puntos, hay 9 regiones, destacando Extremadura con una nota de 7.64. La dispersión en estos resultados entre las distintas CCAA es elevada con una desviación típica cercana a 1.

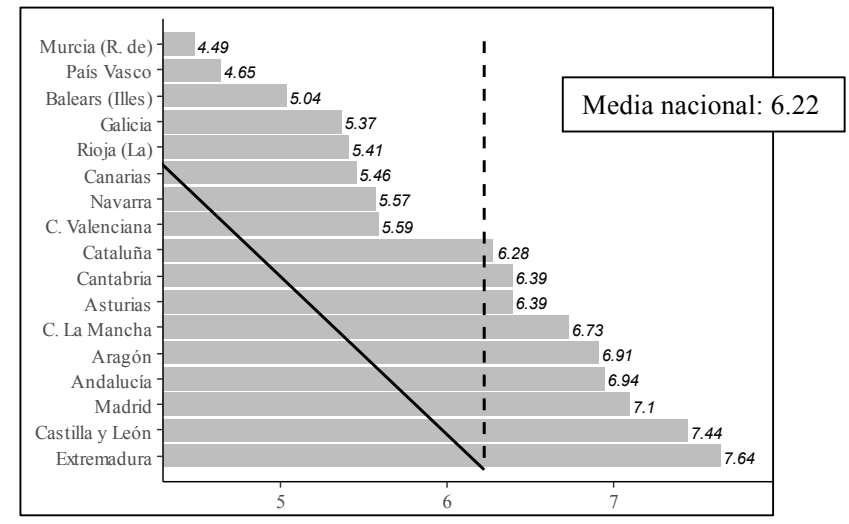

Figura 4. Nota media de MACSII en 2018 en la convocatoria ordinaria 
Al comparar las calificaciones medias de la Selectividad y de la materia MACSII (figura 5) por CCAA en el año 2018 se observa la gran variabilidad existente entre las notas de la materia en contraposición a la mayor estabilidad de la media de toda la prueba conjunta.

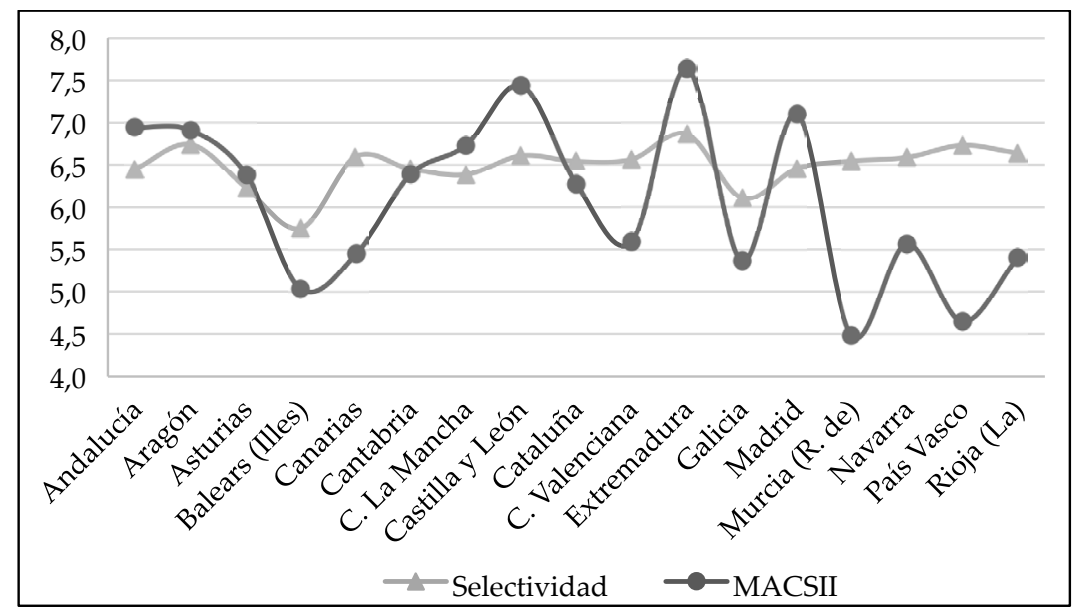

Figura 5. Nota media Selectividad versus MACSII en 2018

En la figura 6 se pueden observar las notas de Selectividad frente a las de Matemáticas CCSSII con una línea que muestra la tendencia inherente a los mismos, reflejando las bandas grises el intervalo de confianza de dicho ajuste al 95\%. Todas las CCAA se mantienen dentro de los límites del intervalo salvo Baleares y Galicia.

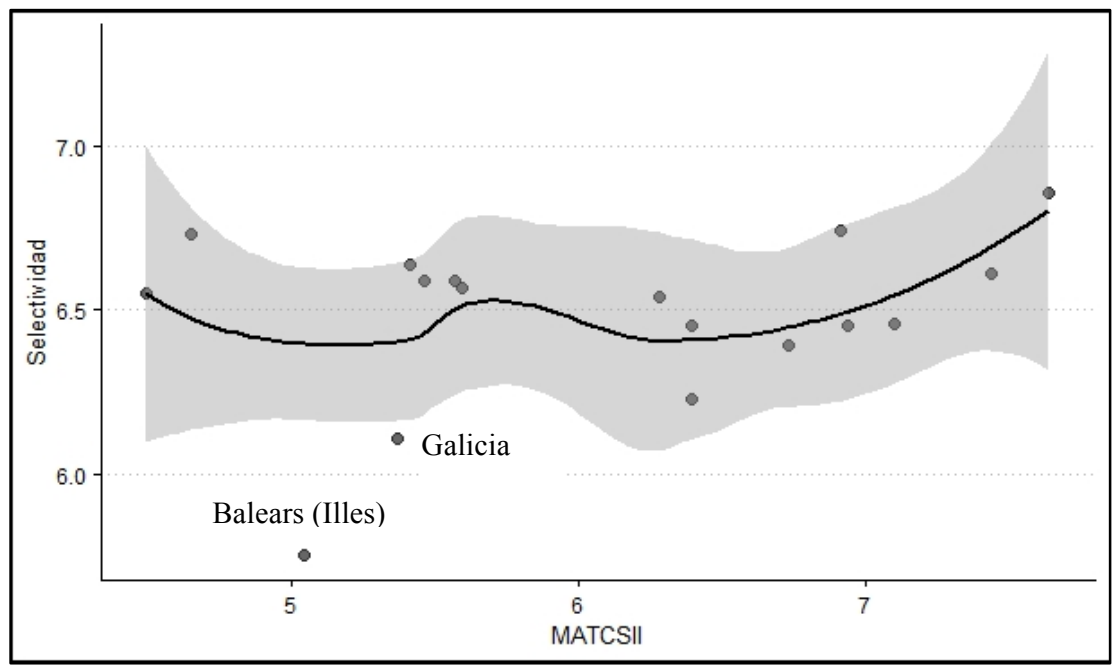

Figura 6. Nota media Selectividad versus MACSII en 2018 
Los resultados de matemáticas en 2018 por tramos de calificaciones se recogen en la figura 7. El porcentaje de alumnos que no ha superado la Selectividad es mayor en la Región de Murcia y en el País Vasco alcanzando más del 50\%. Los estudiantes extremeños, por el contrario, son los que han suspendido en una menor proporción, solo el $7.8 \%$, le siguen los de Castilla y León con un $10.7 \%$. Cabe reseñar que es este mismo alumnado el que alcanza unas calificaciones más elevadas.

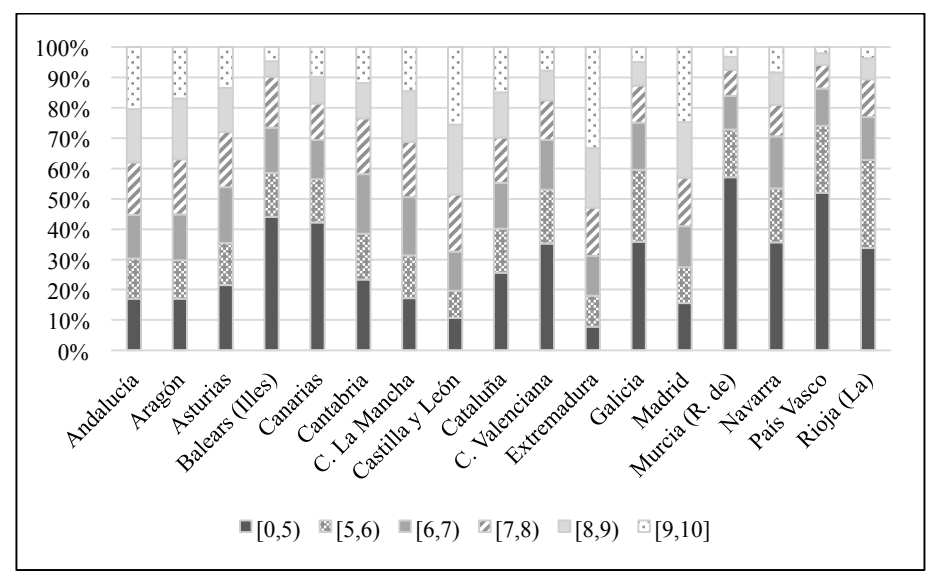

Figura 7. Distribución de las calificaciones de MACSII por tramos en 2018

Analizando detenidamente la distribución de los porcentajes de aquellos estudiantes con una calificación igual o superior a 9 puntos (figura 8), se remarca la diferencia existente entre las regiones. En el País Vasco solamente el 1.96\% de los estudiantes obtienen esta calificación mientras que en Extremadura se llega hasta el 33.38\%.

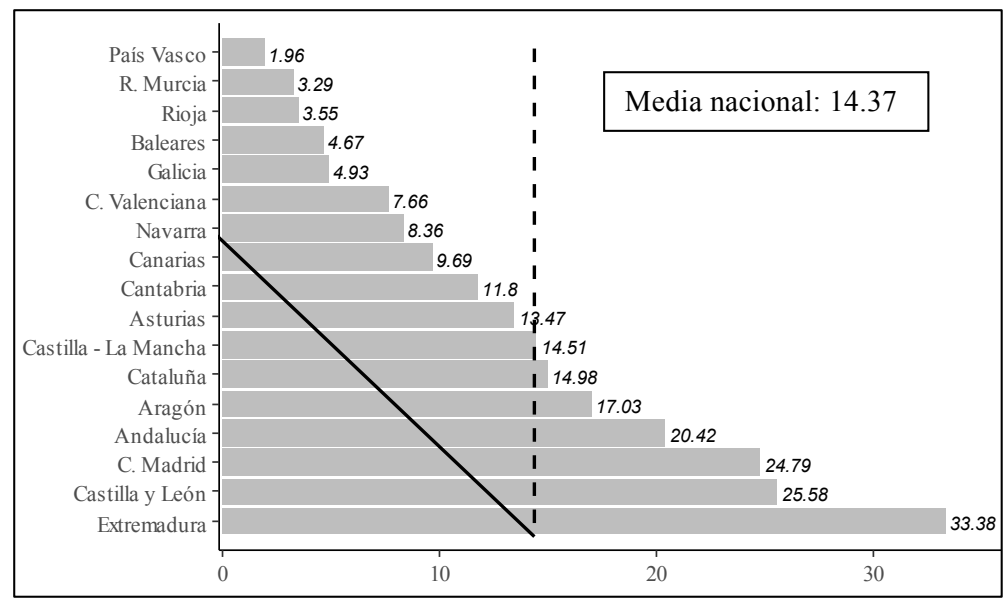

Figura 8. Porcentaje de estudiantes con una calificación igual o superior a 9 en MACSII en 2018 


\section{Valoración del panel de expertos}

Se ha analizado, en primer lugar, la fiabilidad de las puntuaciones de los expertos, obteniendo un valor del alpha de Cronbach de 0,798 , lo que indica que existe una fuerte consistencia interna entre el grado de dificultad observado. Además, con las valoraciones realizadas se ha comprobado que no existe mucha discrepancia entre ellas, pues la dispersión del grado de dificultad ha estado, en cada región en torno a 1 punto, salvo en el caso de Aragón, la Comunidad de Madrid y La Rioja que está alrededor de 2 puntos. En Navarra la representatividad de la valoración media es muy alta al tener un coeficiente de variación muy cercano a 0 . En la figura 9 se recogen, junto con la nota media en la materia de MACSII de cada CCAA, el grado de dificultad estimado por los profesores que han formado el panel de expertos.

Los exámenes valorados con una menor dificultad han sido los de Extremadura, Castilla y León, y Andalucía, siendo estas comunidades las que han obtenido unos de los mejores resultados en la prueba. La Comunidad de Madrid, con una media de 7.10 puntos, sin embargo, presenta un grado de dificultad medio. Según el panel de expertos, los exámenes de La Rioja y del País Vasco han sido los más difíciles en esta convocatoria, teniendo estas regiones unas de las puntuaciones más bajas en la prueba. En la Región de Murcia la nota media fue bastante baja (4.49) pero consideran que presentaba un nivel de dificultad medio, lo que no justifica esta calificación.

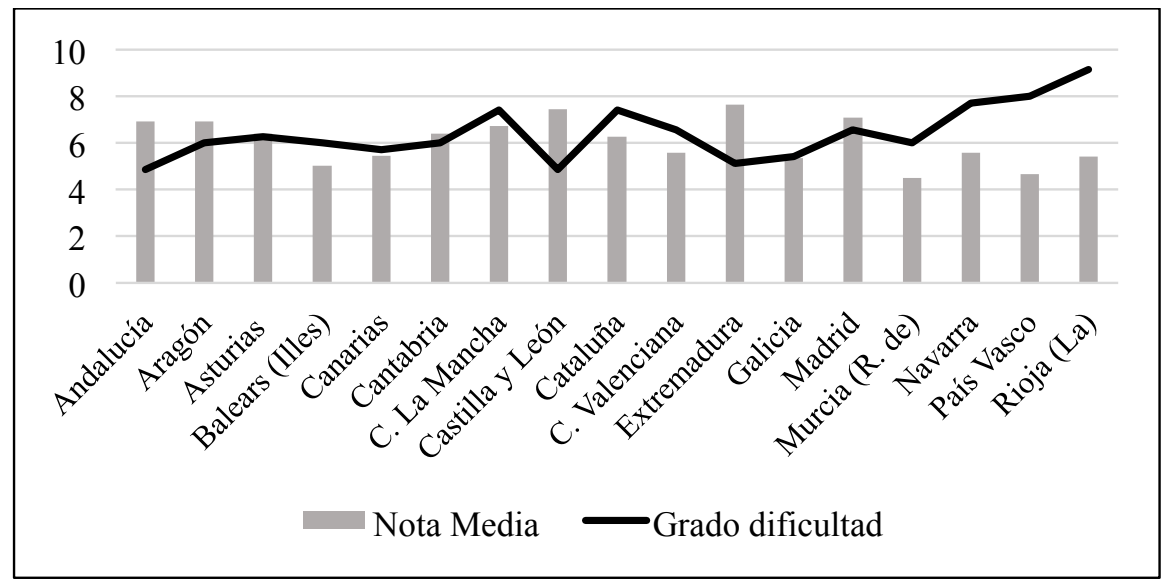

Figura 9. Grado de dificultad establecido por un panel de expertos y nota media de la materia. Convocatoria ordinaria 2018

Se ha calculado el coeficiente de correlación de Pearson entre la nota media y el grado de dificultad establecido por el panel de expertos. Éste alcanza un valor de -0.414 (estadísticamente relevante al 95\% del nivel de confianza, p-valor $<0,05$ ) lo que, de alguna manera, evidencia que el grado de dificultad puede influir en la calificación final. 


\section{Discusión y Conclusiones}

La prueba de acceso a la Universidad es un examen escrito que realizan los estudiantes que quieren comenzar alguno de los grados que ofertan anualmente las Universidades, ya sean públicas o privadas, de España. Su puntuación es importante ya que les puede permitir acceder a uno u otro. El alumnado debe tomar una de las decisiones más importantes de su vida como es la elección de la carrera a estudiar que le conduzca a su futura profesión.

El objetivo de esta prueba es valorar de manera objetiva, la madurez académica del estudiante, así como los conocimientos y capacidades adquiridos en el Bachillerato, que confirmen que han alcanzado las competencias necesarias para afrontar con éxito los estudios universitarios.

Se realiza, durante tres días, en cada Comunidad Autónoma, al finalizar el segundo curso de bachillerato, y su estructura y organización depende de ella. El contenido de las mismas es bastante similar ya que recogen al menos el 70\% del currículo de Bachillerato aprobado en las matrices de especificaciones establecidas en el BOE (2016). Sin embargo, se ha observado en la revisión realizada en este trabajo, que la composición del examen, tipo de preguntas y la valoración de las cuestiones planteadas difiere entre regiones. Incluso la distribución diaria de materias es diferente (lo que puede condicionar la actitud y el rendimiento del alumnado ante el examen de determinadas asignaturas) al igual que la denominación de la prueba.

Todas estas diferencias han podido influir en los resultados alcanzados por el alumnado presentado a la prueba. En la convocatoria ordinaria de la Selectividad en el año 2018, el 93.29\% de los estudiantes aprobaron en todo el territorio nacional, aunque la distribución por Comunidades es dispar, varía desde el 89.93\% de aprobados en Galicia al $97.90 \%$ del País Vasco. La nota media alcanzada tampoco es homogénea, Extremadura es la región con la mayor puntuación, cercana al 7, mientras que los estudiantes de Baleares sólo llegaron al 5.75 de nota media.

En la materia MACSII también se han encontrado importantes variaciones. La media nacional alcanzada ha sido de 6.22 puntos, pero existe una brecha de 3.15 puntos entre la mayor (Extremadura con un 7.64) y la menor calificación (la Región de Murcia con un 4.49), estando la desviación típica cercana a 1. Castilla León y Madrid superan los 7 puntos de media, mientras que Baleares y el País Vasco están en el entorno del 5.

Los resultados de esta materia por tramos de calificaciones siguen evidenciando las divergencias entre las distintas CCAA. En las mayores puntuaciones (tramo comprendido entre 9 y 10 puntos) estas diferencias aumentan: frente al $33.38 \%$ de estudiantes extremeños que alcanza esta calificación se encuentran solamente el $1.96 \%$ del País Vasco.

Ante toda esta variabilidad se ha recurrido a un panel de expertos, formados por profesores relacionados con la materia y que han formado parte del equipo de correctores de Selectividad, para que analicen el grado de dificultad de los exámenes propuestos en cada CCAA. Han concluido que los exámenes más difíciles de la convocatoria ordinaria de 2018 son el de La Rioja y el del País Vasco, siendo sus calificaciones medias de las más bajas de la asignatura. Los de menor dificultad se corresponden con 
aquellas regiones que alcanzaron las máximas puntuaciones en la prueba (Extremadura, Castilla y León y Andalucía). Se constata que el grado de dificultad puede influir en la calificación media obtenida en cada región.

Entre las limitaciones de este trabajo está el ser un estudio descriptivo en el que se refleja una situación determinada. Por otro lado, se basa solo en una de las asignaturas de la selectividad, por lo que las conclusiones podrían no ser extrapolables a otras. Lo que sí podemos afirmar, es que desde la óptica de la asignatura MATCSII, se han detectado diferencias en la Selectividad entre las distintas CCAA, no sólo en los resultados, sino también en la estructura.

En una línea futura de investigación se quiere extender este estudio a otras materias incluidas en la prueba de acceso y ver la trayectoria temporal de los resultados en todas ellas. Además, se quiere utilizar taxonomías para analizar la estructura de conocimiento subyacente a las preguntas y conceptos del examen (como puede ser la de Bloom et al., 1956) y de esta forma analizar si las habilidades adquiridas por los estudiantes de cada región son las mismas.

Para garantizar la igualdad en los contenidos y en los criterios de evaluación se ha planteado la posibilidad de hacer una única prueba a nivel nacional, hecho pendiente de discutir en el seno del Ministerio de Educación y Formación Profesional. Los Rectores de las distintas Universidades han reclamado la creación de una comisión para mejorar la prueba y están pendientes de su formación. Una medida intermedia, que podría tener una buena aceptación y que conduciría a evitar disparidades es la coordinación nacional entre los distintos responsables de materias que pudiera establecer las bases mínimas para que todos los exámenes fueran lo más homogéneos posible.

\section{Referencias}

Bernad J.A. y Escudero, T. (1978). Pruebas de acceso a la universidad y su relación con los rasgos de madurez del alumno. Revista de Educación, no 258-259, 56-89.

Bloom, B. S., Englehart, M. D., Furst, E. J., Hill, W. H. y Krathwohl, D. R. (1956). The Taxonomy of educational objectives, handbook I: The Cognitive domain. New York: David McKay Co., Inc.

Boal Sánchez, N., Bueno García, C., Lerís López, M. D., y Sein-Echaluce Lacleta, M. L. (2008). Las habilidades matemáticas evaluadas en las pruebas de acceso a la Universidad. Un estudio en varias universidades públicas españolas. Revista de Investigación Educativa, 26(1), 11-23. Recuperado a partir de https://revistas.um.es/ rie/article/view/94081

BOE (2015). 03/01/2015. Currículo de Bachillerato (nacional): Real Decreto 1105/2014, de 26 de diciembre (BOE 03/01/2015), por el que se establece el currículo básico de la Educación Secundaria Obligatoria y del Bachillerato.

BOE (2016). 30/7/2016 Evaluación Final de Bachillerato (modificada por el RDL-5/2016): Real Decreto 310/2016, de 29 de julio, por el que se regulan las evaluaciones finales de Educación Secundaria Obligatoria y de Bachillerato. 
BOE (2019). 15/01/2019. Orden ministerial EBAU 2019: Orden PCI/12/2019, de 14 de enero. Recuperado de https://www.boe.es/eli/es/o/2019/01/14/pci12

Contreras de la Fuente, A., Ordóñez L. y Wilhelmi M. (2010). Influencia de las pruebas de acceso a la Universidad en la Enseñanza de la integral definida en el Bachillerato. Enseñanza de la Ciencia, 28(3), 367-384.

Cuxart, A.; Longford, N. (1998): Monitoring the University Admission Process in Spain. Higher Education in Europe, 23(3), 385-396, DOI:10.1080/0379772980230310

Escudero Escorza, T.; Bueno García, C. (1994). Examen de selectividad: El estudio del tribunal paralelo. Revista de educación, 12, 281-298.

Gairin, J.M., Muñoz, J.M. y Oller, A.M. (2013). Anomalías en los procesos de identificación de errores en las pruebas escritas de matemáticas de las P.A.U. Campo Abierto, 32(2), 27-50.

Gairin, J.M., Muñoz, J.M. y Oller, A.M. (2012). Propuesta de un modelo para la calificación de exámenes de matemáticas. En A. Estepa, A. Contreras, J. Deulofeu, M.C. Penalva, F.J. García y L. Ordoñez (Eds.). Investigación en Educación Matemática XVI (261-274 pp.). Jaén: SEIEM.

Gaviria, J.L. (2005). La equiparación del expediente de bachillerato en el proceso de selección de alumnos para el acceso a la Universidad. Revista de Educación, 337, 351-387.

Grau, R., Cuxart, A. y Martí Recober, M. (2002). La calidad del proceso de corrección de pruebas de acceso a la universidad: variabilidad y factores. Revista de Investigación Educativa, 20 (1), 209-223.

Lorenzo Moledo, M., Argos González, J., Hernández García, J. y Vera Vila, J. (2014). El acceso y la entrada del estudiante a la universidad: Situación y propuestas de mejora facilitadoras del tránsito. Educación XX1, 17 (1), 15-38. doi:10.5944/educxx1.17.1.9951.

Mengual, E., Albarracín, L., Muñoz-Escolano, J. M., Oller-Marcén, A. M. y Gorgorió, N. (2019). Diseño de criterios para reducir la variabilidad en la calificación de exámenes de matemáticas en pruebas de acceso a la universidad. PNA- Revista de Investigación en Didáctica de la Matemática 13(2), 62-83.

Muñoz-Repiso Izaguirre, M. y Murillo Torrecilla, F.J. (1997). Los resultados en la selectividad actual: algunas cuestiones a debate. Revista de Educación, 314, 29-48.

Muñoz-Repiso, M.; Muñoz, F.; Palacios, C. y Valle, J. (1991). Las calificaciones en las pruebas de aptitud para el acceso a la Universidad. Centro de Investigación, Documentación y Evaluación C.I.D.E., Madrid.

Nortes Martínez-Artero, R., Nortes Checa, A. y Lozano Pato, F. (2015). Las correcciones en Matemáticas en las Pruebas de Acceso a la Universidad. Education Siglo XXI, 33(3), 199-222.

Rodríguez-Muñiz, L.J., Díaz, P., Mier, V. y Alonso, P. (2016). Washback Effect of University Entrance exams in Applied Mathematics to Social Sciences. PLoS one, 11(12), e0167544. doi: http://dx.doi.org/10.1371/journal.pone.0167544.

Ruiz de Gauna Gorostiza, J., Sarasua Fernández, J. y García Iturrioz, J.M. (2011). Una tipología y clasificación de los ejercicios de matemáticas de selectividad. Epsilón Revista de Educación Matemática, 28(2), 78, 21-38. 
Ruiz de Gauna Gorostiza, J.R., Dávila Balsera, P., Etxeberria Murgiondo, J. y Sarasua Fernández, J.M. (2013) Pruebas de selectividad en Matemáticas en la UPV-EHU. Resultados y opiniones de los profesores. Revista de Educación, 362, 217-246. doi: http://dx.doi.org/10.4438/1988-592X-RE-2011-362-159.

Ruiz-Hidalgo, J.F, Herrera Beltrán, M.E. y Velasco M.V. (2019). Tareas de Cálculo en las pruebas de acceso a la Universidad. Revista de Educación, 386, 137-164. doi: http:// dx.doi.org/ 10.4438/1988-592X-RE-2019-386-430.

Fecha de recepción: 27 de abril de 2020.

Fecha de revisión: 7 de mayo de 2020.

Fecha de aceptación: 10 de diciembre de 2020. 